\title{
AVANÇOS E PERSPECTIVAS DA ATUAÇÃO DO ENFERMEIRO EM ESTRATÉGIA SAÚDE DA FAMILIA
}

Rosiane Filipin Rangel¹, Mônica de Melo Fugali², Dirce Stein Backes ${ }^{3}$, Maria Helena Gehlen ${ }^{4}$, Martha Helena Teixeira de Souza ${ }^{5}$

RESUMO: Objetivou-se, com este estudo, compreender o significado da atuação do enfermeiro em uma equipe da Estratégia Saúde da Família. Trata-se de uma pesquisa qualitativa exploratória realizada com uma equipe da Estratégia Saúde da Família que integra uma Unidade Básica de Saúde. Os dados foram coletados por meio de entrevista e analisados por meio da análise de conteúdo, sendo que dessa análise emergiram cinco categorias. Os resultados evidenciam o papel do enfermeiro como sendo de importância, pois esse é considerado o ponto de referência da Estratégia. A Estratégia está contribuindo para o viver saudável do indivíduo/família, por meio das orientações e informações de promoção da saúde e de prevenção de doenças. Conclui-se que se deve fazer uma reavaliação das políticas públicas em saúde na tentativa de minimizar as dificuldades encontradas.

PALAVRAS-CHAVE: Saúde da família; Enfermagem; Sistema único de saúde; Pesquisa em enfermagem.

\section{ADVANCES AND PERSPECTIVES OF THE NURSE'S ROLE IN THE FAMILY HEALTH STRATEGY}

ABSTRACT: The aim of this study was to understand the meaning of the nurse's role in a Family Health Strategy team. The research is qualitative and exploratory, and was undertaken by a Family Health Strategy team in a Basic Health Unit. The data was collected by interview and analyzed by content analysis, with five categories emerging. The results provide evidence that the role of the nurse is important, as this is considered the reference point of the Strategy. The strategy is contributing to healthy living in individuals/families, by means of advice, health promotion and prevention of disease. It is concluded that public policies should be re-evaluated as an attempt to minimize the difficulties encountered.

KEYWORDS: Family health; Nursing; Unified health system; Nursing research.

\section{AVANCES Y PERSPECTIVAS DE LA ACTUACÓN DEL ENFERMERO EN ESTRATEGIA SALUD DE LA FAMILIA}

RESUMEN: El objetivo con este estudio fue comprender el significado de la actuacción del enfermero en un equipo de Estrategia Salud de la Familia. Este es un estudio de investigación cualitativa exploratoria realizado con un equipo de Estrategia Salud de la Familia que integra una Unidad Básica de Salud. Los datos fueron recogidos por medio de entrevista y analizados por medio del análisis de contenido. De ese análisis surgieron cinco categorías. Los resultados evidencían la importancia del papel del enfermero, pues este es considerado el punto de referencia de la Estrategia. La Estrategia contribuye para el vivir saludable del individuo/familia, por medio de las orientaciones e informaciones de promoción de la salud y de prevención de enfermedades. Se concluye que es necesario hacer una reevaluación de las políticas públicas en salud en la tentativa de minimizar las dificultades encontradas.

PALABRAS-CLAVE: Salud de la família; Enfermería; Sistema único de salud; Investigación en enfermería.

\footnotetext{
${ }^{1}$ Enfermeira. Mestre em Enfermagem. Professora do Curso de Graduação em Enfermagem do Centro Universitário Franciscano UNIFRA. Membro do Grupo de Estudos e Pesquisa em Empreendedorismo Social na Enfermagem e Saúde - GEPESES.

${ }^{2}$ Enfermeira. Especializanda em Enfermagem do Trabalho pelo Sistema Educacional Galileu.

${ }^{3}$ Enfermeira. Doutora em Enfermagem. Professora do Curso de Graduação em Enfermagem do UNIFRA. Líder do GEPESES.

${ }^{4}$ Enfermeira. Mestre em Educação. Professora do Curso de Graduação Enfermagem da UNIFRA. Especializanda em Educação Inclusiva e Psicopedagogia Membro do GEPESES.

${ }^{5}$ Enfermeira. Mestre em Geomática. Professora do Curso de Graduação em Enfermagem do UNIFRA. Doutoranda pelo Programa de Pós-Graduação em Enfermagem da Universidade Federal de São Paulo. Membro do GEPESES.
} 


\section{INTRODUÇÃO}

A partir da Reforma Sanitária, o conceito de saúde ganhou novos significados e passou a assumir uma concepção mais concreta e complexa e que se define a saúde como um bem público, inerente à cidadania, que deve ser garantido por direito a todo e qualquer cidadão brasileiro, embora apresente limitações burocráticas ${ }^{(1)}$.

Frente a isso, e a fim de atingir a compreensão ampliada e complexa de saúde, foram necessárias estratégias capazes de compreender o ser humano em seu contexto familiar, ou seja, inserido em sua realidade concreta. Dentre as iniciativas, destaca-se a Estratégia Saúde da Família (ESF), inicialmente denominada Programa Saúde da Família (PSF). Esse foi implantado em 1994, pelo Ministério da Saúde, na tentativa de reverter o modelo assistencial vigente, até então hegemônico e centrado em ações fragmentadas, indivisíveis e com o foco na doença ${ }^{(2)}$.

Visando melhorar as condições de saúde da população, a partir de um novo modelo de intervenção com foco na promoção, proteção, diagnóstico precoce, tratamento e recuperação da saúde, a ESF observa os princípios, diretrizes e doutrinas do Sistema Único de Saúde (SUS) em todo o território nacional, com ações voltadas às famílias e comunidade $^{(3)}$. Nessa busca por melhores práticas em saúde, o então PSF deixou de ser um programa vertical, de caráter governamental e passou a ser uma política de governo, denominada $\operatorname{ESF}^{(4)}$.

Nessa direção, foram criadas equipes de ESF, cada uma responsável por aproximadamente 800 famílias, residentes em território urbano e rural, compostas normalmente por: um médico generalista, dois enfermeiros, um técnico em enfermagem e quatro a seis agentes comunitários de saúde. A partir de um esforço governamental para a melhoria destas intervenções, foi integrado também um profissional odontólogo, para contemplar a atenção à saúde bucal ${ }^{(5)}$.

O enfermeiro, neste campo estratégico de conquistas, tem como função supervisionar e qualificar a equipe de enfermagem e os agentes comunitários de saúde, bem como realizar o cogerenciamento da unidade ${ }^{(6)}$. Nessa lógica, o enfermeiro passa a assumir importantes funções, dentre outras, como educador, prestador de cuidados, consultor, auscultador/identificador dos problemas da comunidade, articulador, integrador, planejador e interlocutor político, dando formas variáveis no eixo central da construção do trabalho ${ }^{(6-7)}$.
Na atuação comunitária, o enfermeiro deve promover e preservar a saúde da população, necessitando competências para decidir, viabilizar intervenções compatíveis com aspectos ético-legais e estabelecer ligações entre o cuidado de enfermagem ao indivíduo, família e grupos com seu contexto( ${ }^{(8)}$.

Diante das novas diretrizes curriculares para a formação em Enfermagem e dos desafios gerenciais do SUS que emergem se fazem necessários novos debates, estudos e investigações, a fim de possibilitar outros olhares ereferenciais capazes de atender aos princípios da transversalidade e integralidade das ações de saúde/cuidado ${ }^{(9)}$.

Destarte, o enfermeiro necessita abrir caminhos para novos olhares de atuação profissional, no sentido de superar as práticas assistencialistas, ultrapassar normas e rotinas inflexíveis e avançar para além do cuidado pontual e unidimensional. Para tanto ele adota novas abordagens de intervenção em saúde, a partir de práticas educativas criativas e inovadoras de cuidado em Enfermagem/saúde e voltadas para a promoção e educação em saúde, no sentido de possibilitar a ampliação da rede de interações e associações de cuidado em saúde. E, dessa forma, pode promover a participação dos usuários como autores da sua própria história ${ }^{(9-10)}$.

Diante do exposto, objetivou-se com essa pesquisa compreender o significado da atuação do enfermeiro em uma equipe da Estratégia Saúde da Família.

\section{METODOLOGIA}

O presente estudo caracteriza-se como uma pesquisa qualitativa, de cunho descritivo-exploratório. A pesquisa qualitativa é aquela capaz de incorporar a questão do significado e da intencionalidade como inerentes aos atos, às relações e às estruturas sociais, sendo essas últimas, desde a sua criação, e na sua transformação, entendidas como construções humanas significativas ${ }^{(11)}$.

A introdução dessa definição incluiu consequências teóricas e práticas na abordagem social. Uma delas foi a interrogação sobre ser ou não ser considerado científico um trabalho de investigação sobre os níveis mais profundos das relações sociais e que não pudesse operacionalizar os resultados em números variados, já que estes foram os critérios geralmente aceitos como científicos para emitir juízo de verdade no campo intelectual ${ }^{(11)}$. A fase exploratória da pesquisa possibilitou ser um começo da escolha do tópico de investigação, de delimitação do problema, de definição do objeto e dos objetivos, de construção do marco teórico conceitual, dos instrumentos de coleta de dados e da exploração do campo ${ }^{(11)}$. 
O estudo foi realizado em uma Unidade Básica de Saúde (UBS) na região oeste da Cidade de Santa Maria-RS, e cuja equipe é composta por um enfermeiro, um médico, um técnico em enfermagem, quatro agentes de saúde, uma secretária e uma funcionária dos serviços gerais.

Os dados foram coletados no período de fevereiro a abril de 2010, por meio de entrevista com questões norteadoras. A observação complementou com dados de identificação e outros aspectos que puderam facilitar a análise e a determinação das categorias, como por exemplo, a expressão facial, o olhar e outros ${ }^{(11)}$. As entrevistas foram realizadas em dias e horários previamente agendados, gravadas e transcritas.

Os dados foram analisados por meio da análise de conteúdo, na qual a meta foi a descrição objetiva, sistemática e qualitativa do conteúdo evidente da comunicação. Esta análise visou o conhecimento de variáveis de ordem psicológica, sociológica e histórica, por meio de conclusões baseadas em indicadores reconstruídos a partir de entrevistas particulares ${ }^{(12)}$.

Os sujeitos do estudo foram 8 profissionais da equipe da ESF da referida UBS, e 12 usuários da mesma, totalizando 20 entrevistados. Adotaram-se como critérios de inclusão pertencer à equipe da ESF ou ser usuário desta, e ter idade igual ou superior a 18 anos além de assinar o Termo de Consentimento Livre e Esclarecido.

Para atender os critérios éticos, foram observadas as recomendações da Resolução do Conselho Nacional de Saúde 196/96, a qual prescreve a ética na pesquisa com os seres humanos, sendo assegurado o sigilo e o anonimato dos sujeitos envolvidos, e observando a não-manipulação dos dados para outros fins ${ }^{(13)}$. Foram analisadas questões relacionadas à solicitação de autorização a direção da instituição onde foi realizada a pesquisa. O projeto foi aprovado pelo Comitê de Ética em Pesquisa do Centro Universitário Franciscano - UNIFRA, sob o número 333/2008. As falas dos participantes foram identificadas, ao longo do texto, pelas letras "P" (profissionais da estratégia) e "U" (usuário), seguidas de um número, correspondente à ordem das falas.

\section{RESULTADOS}

O processo de análise dos dados resultou nas seguintes categorias: $O$ significado da atuação do enfermeiro na ESF; Compreendendo o vínculo profissional de saúde - usuário; A contribuição da ESF para o viver saudável; Avanços relacionados à ESF; Dificuldades relacionadas à $E S F$, cujo conteúdo são descritos a seguir.

\section{O significado da atuação do enfermeiro na ESF}

Nas respostas dos profissionais, observou-se que estes consideram o enfermeiro como aquele que coordena, supervisiona, lidera a ESF; no entanto, crêem que esse profissional deve acreditar mais na iniciativa da Estratégia. Eles reconhecem que, sem o enfermeiro, a Estratégia não é efetiva nas visitas domiciliares, pois é ele que materializa o processo de cuidado, acolhe o usuário, tem participação dentro da família, compreende as necessidades, escuta e é o ponto de referência, o "testa de ferro".

O enfermeiro é como se fosse à porta de entrada sabe? E, o médico parece sempre tão gelado atrás da mesa! Tu vai falando dos teus problemas e ele só passa a medicação, a orientação. O enfermeiro não, ele já te entende mais como se fosse da família. Quase sempre o pessoal vem aqui e diz: "ah, eu quero falar com a enfermeira tal". Às vezes, é um problema de familia, dai procura aquela pessoa, por saber que ela vai te escutar, te aconselhar. (P7)

Na opinião dos usuários, o significado da atuação do enfermeiro na ESF é de grande importância. Eles também ressaltam que, sem a presença desse profissional, muitas atividades não seriam desenvolvidas.

O enfermeiro é muito importante, pois se precisa de alguém antes, para depois passar para o médico. Eles ajudam! É necessário um enfermeiro aqui no posto. (U12)

\section{Compreendendo o vínculo profissional de saúde - usuário}

Os profissionais acreditam que o vínculo se constroi através do agente de saúde, pelo fato desse membro da equipe conhecer a realidade da comunidade, fazer as visitas domiciliares, as orientações, o acolhimento, a aproximação, o chamar pelo nome, do agir como se fosse da família, de interessar-se pela comunidade, de inspirara ética, a segurança e a confiança.

Quem faz o vínculo é o agente de saúde! O enfermeiro é o principal elo que existe entre o agente de saúde, família e Estratégia Saúde da Família. O enfermeiro é tudo, 90\% dos problemas da população quem resolve é ele. (P8)

Para o usuário, o vínculo se consolida através de uma boa comunicação, quando o enfermeiro cria uma empatia por meio do acolhimento, do respeito, do carinho, da atenção e, assim, acaba passando segurança e confiança para 
a comunidade. Ele acredita, ainda, que para se formar o vínculo é importante que tanto o enfermeiro como toda a equipe transmitam confiança e segurança, o que o faz se sentir mais tranquilo e estimulado a buscar a UBS.

Eu tenho um carinho, um respeito pela enfermeira. Ela, como toda a equipe de saúde, está aqui para nos ajudar. Eu converso, falo um pouco sobre minha vida. Até para se formar um vínculo tem que ter uma certa intimidade. (U12)

\section{A contribuição da ESF para o viver saudável}

No entendimento dos profissionais, a ESF está contribuindo para o viver saudável do indivíduo/família, por meio das orientações e informações de promoção da saúde e de prevenção de doenças fornecidas através das realizações dos grupos, quando se ensina e se reeduca os usuários, oferecendo oportunidades de a comunidade ter acesso aos projetos da Estratégia. Eles também consideram que houve melhoria nos indicadores de saúde:

Os próprios índices das politicas do município já mostram que a mortalidade infantil caiu. Os idosos estão vivendo uma vida mais saudável, os hipertensos e diabéticos têm o acompanhamento dos enfermeiros dentro da casa da família para dar orientações, está melhorando muito. A estratégia veio para realmente resolver o problema da comunidade. $(\mathrm{P} 2)$

A contribuição da ESF no viver saudável do indivíduo/ família, para os usuários, ocorre com a realização dos grupos do "Hiperdia", puericultura e de gestantes, onde os profissionais sempre trazem informações e orientações sobre as medicações, alimentação, exercícios, as vacinas que ainda não foram feitas, e sobre a amamentação.

\section{Avanços relacionados à ESF}

O crescimento na procura, pelos usuários, dos serviços de saúde, é considerado, na fala dos profissionais da saúde, como um dos maiores avanços da ESF:

Diminuiu a mortalidade infantil, como também a do idoso. Os usuários estão buscando uma melhor qualidade de vida realmente, a Estratégia está dando certo. (P2)

Os usuários da ESF consideram como principais avanços em saúde: a existência de uma Unidade de Saúde na comunidade; $\mathrm{o}$ ambiente $\mathrm{e} o$ atendimento dos profissionais mudaram para melhor; a vinda de mais medicações para a Unidade; existência das visitas domiciliares, tanto do enfermeiro como do próprio médico, principalmente aos usuários que têm dificuldade de se locomover até às Unidades Básicas; eles recebem mais informações sobre o que está acontecendo com sua saúde; o horário de atendimento da Unidade também melhorou:

Antes, era só uma sala, e hoje temos um lugar adequado para consultar. Antes, atendiam a gente no meio de todo mundo e todos ficavam olhando. (U7)

\section{Dificuldades encontradas na atuação do ESF}

$\mathrm{Na}$ fala dos profissionais, evidencia-se que esses acreditam que as principais dificuldades encontradas na ESF referem-se à estrutura física, à falta de um veículo disponível para a Unidade e de mais profissionais de saúde (médico, enfermeiro, técnico em enfermagem e odontólogo), número insuficiente de fichas distribuídas na Unidade, falta de medicamento e de segurança no posto e inviabilidade do horário de atendimento clínico, pois há dias em que se atende por somente um turno:

A falta do médico que, muitas vezes, a enfermeira deixa de realmente cumprir o papel que é dela pra fazer outras coisas. Acaba deixando de ter aquela relação com a familia, no dia a dia. E outra dificuldade grande que eu vejo é que muitas áreas estão sem agente e o pessoal não tem acesso até o posto, porque não tem informação. O usuário acaba tendo, assim, uma maneira desencontrada de buscar uma qualidade melhor de vida. (P2)

Assim, como evidenciado nas falas dos profissionais, os usuários também acreditam que as principais dificuldades encontradas na atuação do ESF estão relacionadas ao número insuficiente de fichas que são distribuídas na Unidade; à falta de profissionais da saúde como médico, odontólogo, agente de saúde, enfermeiro e técnico em enfermagem; às medicações que, na maioria, estão em falta; e também à inexistência de um veículo disponível na Unidade para transportar os usuários até o Pronto Atendimento Municipal ou ao Hospital Universitário quando preciso, ou para aqueles que estão impossibilitados de se dirigir até à Unidade; e por último, a falta de segurança na Unidade:

Vou dar um exemplo da minha mãe. No caso, é muito dificil de conseguir um transporte para levar ela a fazer tratamento, lá no universitário[Hospital]. Tanto os acadêmicos como o enfermeiro, o médico e as agentes de saúde, às vezes, têm muita dificuldade de conseguir. (U10) 


\section{DISCUSSÃO}

Nos resultados, ficou evidente que a atuação do enfermeiro na ESF para os profissionais e usuários é muito importante, mas é indispensável que esse profissional acredite na iniciativa e tenha comprometimento com a comunidade, pois o mesmo é o ponto de referência da Estratégia.

O enfermeiro, em sua prática profissional, precisa atender basicamente a dois aspectos: o de preservar, respeitar e reconhecer a particularidade, individualidade e a variabilidade das situações e necessidades dos usuários; e o de estar em conformidade com determinadas regras e regulamentos, além de se inserir/ integrar permanentemente às atividades da equipe multiprofissional. Para isso, ele deve utilizar algumas estratégias como a visita domiciliar, a educação em saúde e o acolhimento, além de usar alguns instrumentos que permitam reconhecer as características da família como um todo, tais como: genograma e o ecomapa, dentre outros ${ }^{(14)}$.

O enfermeiro desempenha um papel estratégico na formação do sistema de cuidados, entendido como aquele que agrega vários saberes, por ter capacidade de interagir abundantemente com os demais profissionais da equipe da saúde. Nesse entendimento, o enfermeiro gerencia os conhecimentos referentes ao desenvolvimento do trabalho assistencial de enfermagem, colocando em prática sua autonomia para avaliar necessidades assistenciais do usuário, decidindo sobre tipo o cuidado ${ }^{(15)}$.

Os profissionais acreditam que, além do enfermeiro, o agente de saúde é de grande importância na ESF, pois ele conhece a realidade da comunidade. Assim, entende-se que esse profissional tem mais habilidade em levar os usuários para dentro da Estratégia.

$\mathrm{O}$ agente comunitário de saúde merece destaque, pois ele tem contato direto com a comunidade, tendo como função: a realização do mapeamento de sua área de atuação; o cadastramento e acompanhamento das famílias que residem em seu território; a identificação de situações de risco; o desenvolvimento de ações básicas de saúde como a de prevenção, promoção, educação e mobilização comunitária por meio de ações de saneamento e a tentativa de melhorar o meio ambiente; estimular a formação de conselhos locais de saúde, orientando-os a procurar a ESF para receber assessoria em saúde de forma adequada; e participar das atividades, palestras e grupos elaborados pela equipe com o intuito de superar os problemas que são identificados ${ }^{(16)}$.
Salienta-se que o vínculo entre profissional e usuário se constroi através da demonstração de interesse do profissional pela comunidade, da orientação e comunicação entre os mesmos, da confiança, da segurança, do respeito, da atenção, do carinho e da ética. Para muitos, o enfermeiro é o principal elo que existe entre o agente de saúde, família e a ESF, já que, de alguma forma, contribui e participa da vida cotidiana da comunidade, possibilitando ao usuário tranquilidade e segurança para usufruir das atividades e cuidados fornecidos pela equipe de saúde, encorajando-o também a procurar mais seguidamente os serviços oferecidos na Unidade.

O vínculo com os usuários do serviço de saúde se torna eficaz nas ações de saúde e auxilia na participação do usuário na continuidade da procura dos serviços em saúde. Com isso, leva-se em conta que o vínculo ajuda na formação da autonomia dos usuários como também na dos próprios profissionais. Por essa razão, acredita-se que para a criação do vínculo, o usuário deve ser reconhecido como sujeito que fala, julga e deseja ${ }^{(17)}$.

Os profissionais de saúde, muitas vezes, integram as atividades de trabalho com condutas fragmentadas que desfavorecem o crescimento e o desenvolvimento de um novo modelo de saúde para cuidar do coletivo e que seja comprometido com a construção de uma sociedade melhor ${ }^{(18)}$. As ações dispensadas à família parecem ser integrantes de uma prática pautada no modelo biomédico, que não consegue olhar para a mesma e para a comunidade em todas as suas dimensões ${ }^{(18)}$.

Com a multiprofissionalidade, corre-se o risco de agrupar profissionais com diferentes qualificações no mesmo espaço laboral, que trabalham com ênfase na especialidade e autonomia técnica, independentes de um projeto assistencial comum, utilizando-se de pouca comunicação instrumental para interagir ${ }^{(19)}$.

As dificuldades de trabalho na ESF não se limitam à organização multidisciplinar das atividades, mas, também, à sobrecarga de trabalho devido à grande demanda existente. Embora o Ministério da Saúde tenha estabelecido um número de atendimentos, a realidade de algumas equipes apontam demandas muito maiores daquilo que é preconizado ${ }^{(19)}$.

\section{CONSIDERAÇÕES FINAIS}

Com o objetivo de compreender o significado da atuação do enfermeiro em uma equipe da ESF, o estudo realizado permitiu a discussão quanto ao seu papel 
na comunidade, e quanto aos avanços e dificuldades existentes com essa Estratégia.

Percebeu-se que a ESF teve importantes avanços e muitos dos profissionais da saúde já aderiram ao conceito ampliado de saúde, embora outros ainda continuem com o foco centrado no modelo assistencial tradicional.

Diante dos resultados obtidos, acredita-se que a ESF seja uma importante iniciativa, porém, o profissional da saúde precisa ter perfil e estar comprometido com a comunidade, no entando, para isto, sugere-se que essas práticas se iniciem ainda na academia, para despertar, desde cedo, o interesse pela nova concepção em saúde com o foco na promoção da saúde e na educação transformadora para a saúde.

Espera-se que o profissional da saúde esteja realmente empenhado com a comunidade usuária, demonstrando atenção, dedicação, ouvindo e ajudando a melhorar sua qualidade de vida de maneira humanizada.

Além disso, é urgente propor às autoridades que invistam mais nas políticas públicas de saúde existentes, incentivando a sua melhoria com intuito de estimular os profissionais na prática a desenvolver um trabalho digno de cidadania, fazendo com que os usuários aumentem a confiança e a sua participação nos serviços oferecidos pela ESF.

Além dos avanços que a ESF obteve ao buscar a reorganização do modelo existente, deve-se destacar a imposrtância para a reavaliação das políticas públicas em saúde, fomentando a discussão na tentativa de diminuir as dificuldades encontradas.

\section{REFERÊNCIAS}

1. Fleury S. Reforma sanitária brasileira: dilemas entre o instituinte e o instituído. Cienc Saude Colet. 2009;14(3):743-52.

2. Ximenes Neto FRG, Sampaio JJC. Gerentes do território na estratégia saúde da família: análise e perfil de necessidades de qualificação. Rev Bras Enferm. 2007;60(6):687-95.

3. Ministério da Saúde (BR). Secretaria Nacional de Assistência à Saúde. ABC do SUS: doutrinas e princípios. Brasília; 1990.

4. Giovanella L, Mendonça MHM, Almeida PF, Escorel S, Senna MCM, Fausto MCR, et al . Saúde da família: limites e possibilidades para uma abordagem integral de atenção primária à saúde no Brasil. Cienc Saude Colet. [Internet].
2009;14(3) [acesso em 03 set 2009]. Disponível: http:// www.scielosp.org/pdf/csc/v14n3/14.pdf

5. Campos GWS, Minayo MCS, Akerman M, Drukmond Junior M, Carvalho YM. Tratado de saúde coletiva. $2^{\text {a }}$ ed. São Paulo: Hucitec; 2008.

6. Barbosa SP, Aguiar AC. Fatores influentes na permanência dos enfermeiros na estratégia saúde da família em Ipatinga, Minas Gerais, Brasil. Revista APS. 2008;11(4):380-8.

7. Ximenes Neto FRG, Costa FAM, Chagas MIO, Cunha ICKO. Olhares dos enfermeiros acerca de seu processo de trabalho na prescrição de medicamentos na estratégia saúde da família. Rev Bras Enferm. [Internet]. 2007;60(2) [acesso em 08 set 2009]. Disponível: http:// www.scielo.br/pdf/reben/v60n2/a01v60n2.pdf

8. Costa LB, Costa AAS, Saraiva MRB, Barroso MGT. Aplicação de estruturas conceituais na consulta de enfermagem à família. Esc Anna Nery Rev Enferm. [Internet] 2007;11(3) [acesso em 08 set 2009]. Disponível: http://www.scielo.br/pdf/ean/v11n3/v11n3a18.pdf

9. Backes DS, Erdman AL, Lunardi Filho WD, Erdmann RH. Despertando novas abordagens para a gerência do cuidado de enfermagem: estudo qualitativo. Online Braz J Nurs. [Internet] 2009;8(2) [acesso em 10 de set 2009]. Disponível: http://www.objnursing.uff.br/index. php/nursing/article/view/j.1676-4285.2009.2407/527

10. Erdmann AL, Backes MTS, Backes DS, Koerich MS, Baggio MA, Carvalho JN, et al. Gerenciando uma experiência investigativa na promoção do "viver saudável" em um projeto de inclusão social: relato de experiência. Texto \& Contexto Enferm. 2009;18(2):369-77.

11. Bardin L. Análise de conteúdo. 4ª ed. Lisboa: Edições; 2009.

12. Minayo MCS. O desafio do conhecimento: pesquisa qualitativa em saúde. 2a ed. São Paulo: Hucitec; 1993.

13. Ministério da Saúde (BR). Conselho Nacional de Saúde. Diretrizes e normas regulamentadoras de pesquisas envolvendo seres humanos. Resolução n. 196, de 10 out 1996. Brasília:1996.

14. Oliveira RG, Marcon SS. Trabalhar com famílias no programa de saúde da família: a prática do enfermeiro em Maringá-Paraná. Rev Esc Enferm USP. 2007;41(1):65-72.

15. Backes DS, Backes MS, Sousa FGM, Erdmann AL. O 
papel do enfermeiro no contexto hospitalar: a visão de profissionais de saúde. Cienc Cuid Saude. 2008;7(3):319-26.

16. Hildebrand SM, Schimizu HE. Percepção do agente comunitário sobre o programa família saudável. Rev Bras Enferm. 2008;61(3):319-24.

17. Schimith MD, Lima MADS. Acolhimento e vínculo em uma equipe do programa saúde da família. Cad Saude Publica. 2004;20(6):1487-94.

18. Resta DG, Motta MGC. Família em situação de risco e inserção no programa de saúde da família: uma reflexão necessária à prática profissional. Texto \& Contexto Enferm. 2005;14(n.esp):109-15.

19. Oliveira SF, Albuquerque FJB. Programa de saúde da família: uma análise a partir das crenças dos seus prestadores de serviço. Psicol Soc. 2008;20(2):237-46. 